Published in final edited form as:

Future Virol. 2011 April ; 6(4): 421-429.

\title{
HSV-1 ICP0: paving the way for viral replication
}

\author{
Miles C Smith ${ }^{1}$, Chris Boutell ${ }^{2}$, and David J Davido ${ }^{1, \dagger}$ \\ ${ }^{1}$ Department of Molecular Biosciences, University of Kansas, 1200 Sunnyside Avenue, 7047 \\ Haworth Hall, Lawrence, KS 66045, USA \\ ${ }^{2}$ MRC - University of Glasgow Centre for Virus Research, Institute of Infection, Immunity \& \\ Inflammation, 8 Church Street, Glasgow, G11 5JR, Scotland, UK
}

\begin{abstract}
Herpes simplex virus type 1 (HSV-1) has two distinct phases of its viral life cycle: lytic and latent. One viral immediate-early protein that is responsible for determining the balance between productive lytic replication and reactivation from latency is infected cell protein 0 (ICP0). ICP0 is a 775-amino acid really interesting new gene (RING)-finger-containing protein that possesses E3 ubiquitin ligase activity, which is required for ICP0 to activate HSV-1 gene expression, disrupt nuclear domain (ND) 10 structures, mediate the degradation of cellular proteins, and evade the host cell's intrinsic and innate antiviral defenses. This article examines our current understanding of ICP0's transactivating, E3 ubiquitin ligase, and antihost defense activities and their interrelationships to one another. Lastly, we will discuss how these properties of ICP0 may be utilized as possible targets for HSV-1 antiviral therapies.
\end{abstract}

\section{Keywords}

E3 ubiquitin ligase; herpes simplex virus; ICP0; innate immunity; intrinsic defense; nuclear domain 10 ; viral transactivator

\section{The requirement for ICPO during viral replication}

The importance of infected cell protein 0's (ICP0's) role in productive infection and reactivation from latent infections was initially identified from characterizing the replication phenotypes of ICP0-null mutants in cell culture. These early reports showed that ICP0 confers a significant growth advantage during replication, especially at low multiplicities of infection [1-5]. Consistent with cell culture experiments, ICP0-null mutants were subsequently shown to be attenuated for viral growth [5-8] and impaired for viral reactivation when equivalent latent infections were established in vivo [9]. Collectively,

\footnotetext{
(C) 2011 Future Medicine Ltd

${ }^{\dagger}$ Author for correspondence: Tel.: +1 785864 4022, Fax: +1 785864 5294, ddavido@ku.edu.

For reprint orders, please contact: reprints@ futuremedicine.com

Financial \& competing interests disclosure

This work was supported by the Medical Research Council - University of Glasgow Centre for Virus Research (C Boutell) and Public Health Service grant RO1AI72357 from the National Institute of Allergy and Infectious Diseases (DJ Davido). DJ Davido and WP Halford (Southern Illinois University) are co-inventors on a patent application that describes using HSV ICP0 mutants as vaccines to protect against HSV-mediated diseases. The authors have no other relevant affiliations or financial involvement with any organization or entity with a financial interest in or financial conflict with the subject matter or materials discussed in the manuscript apart from those disclosed.

No writing assistance was utilized in the production of this manuscript.
} 
these studies indicated that ICP0 is required for efficient replication and reactivation from latency.

\section{ICP0: a viral transactivator}

One of the first activities described for ICP0 was its ability to activate HSV-1 promoters. Experiments performed more than 20 years ago using transient transfection and reporter assays established that ICP0 is capable of transactivating promoters from all three kinetic classes of HSV-1 genes, including immediate-early, early and late [2,10-14]. Structurefunction studies showed that ICP0's transactivator function stems from regions located both in its $\mathrm{N}$-terminal really interesting new gene (RING)-finger and its C-terminus, which includes a nuclear domain (ND) 10 localization signal $[15,16]$, a dimer/multimer motif $[17,18]$ and a binding site for ubiquitin-specific protease 7 (USP7) (Figure 1) [19]. Deletion or mutation in one or more of these regions impair, to varying degrees, ICP0's ability to fully activate viral gene expression in transient transfection assays and viral infection.

\section{The E3 ubiquitin ligase activity of ICPO}

ICP0 mediates its viral transactivator function, in part, through its $\mathrm{N}$-terminal $\mathrm{C} 3 \mathrm{HC} 4$ zinc $\left(\mathrm{Zn}^{2+}\right)$-binding RING-finger domain (Figure 1), a structural motif that is conserved in all ICP0 alphaherpesvirus orthologs (Figure 2) [20,21]. While initial studies that examined RING-finger domain mutations in ICP0 failed to uncover the biochemical function of this motif, these studies clearly demonstrated the importance of this domain in viral replication. Deletion or point mutations within the RING domain not only affect ICP0's ability to transactivate reporter gene expression [22], but also its ability to stimulate the initiation of lytic infection $[3,23-25]$ and reactivate viral genomes from latency $[6,8,9,26]$. This impairment is clearly observed in mutants deleted for the RING-finger domain or point mutations that disable $\mathrm{Zn}^{2+}$ binding, which display growth phenotype properties equivalent to ICP0-null mutant viruses [3,24].

Insights into the potential biochemical function of ICP0's RING-finger domain came from a number of reports showing that ICP0 could interact with components of the ubiquitinproteasome pathway during infection. These included ICP0's ability to form a strong interaction with USP7 (also known as herpesvirus-associated ubiquitin-specific protease [HAUSP]) [27-29], to induce the degradation of the catalytic subunit of DNA-dependent protein kinase (DNA-PKcs) [30,31], and to disrupt ND10 bodies, leading to the degradation of PML (a core constituent component), its SUMO-modified isoforms and SUMO-modified Sp100 [32-35]. The capacity of ICP0 to induce the degradation of these proteins occurred in a RING-finger- and proteasome-dependent manner, indicating that ICP0 could utilize the ubiquitin-proteasome pathway during viral infection to target specific cellular proteins for degradation. A number of additional cellular proteins have subsequently been identified as being targeted for degradation by ICP0, including the centromeric proteins CENP-A, -B and -C [36-38] and the DNA repair proteins RING-finger protein (RNF)8 and RNF168 [39].

The first evidence demonstrating that ICP0 could act as a viral ubiquitin ligase came from the observation that ICP0 colocalized with conjugated ubiquitin in the nucleus of infected cells $[40,41]$. This attribute was again dependent upon the RING-finger domain of ICP0 and correlated with its transactivator function. ICP0 mutants that displayed reduced abilities or completely failed to colocalize with conjugated ubiquitin were similarly impaired in reporter or viral growth assays [40]. Concurrent with these observations, biochemical studies showed that purified ICP0 had E3 ubiquitin ligase activity in vitro, catalyzing the formation of unanchored polyubiquitin chains in a RING-finger-dependent manner [42]. This activity occurred in an E2-dependent manner, requiring the presence of either UbcH5a (UBE2D1) or UbcH6a (UBE1E1), two highly homologous E2 ubiquitin-conjugating enzymes. No activity 
was seen when other E2 ubiquitin-conjugating enzymes were tested, including $\mathrm{UbcH} 7$ (UBE2L3) and cdc34 (UBE2R1). Consistent with these in vitro observations, UbcH5a and UbcH6a were also shown to co-localize with ICP0 in the nucleus of transfected cells [42], and expression of a catalytically inactive UbcH5a mutant reduced ICP0's ability to degrade PML [43]. These data support the notion that ICP0 actively recruits ubiquitin-conjugating enzymes to target cellular proteins for ubiquitination. A number of recent studies have corroborated the functional importance of ICP0's RING-finger ubiquitin ligase activity during HSV-1 infection. First, the mutation of residues within the RING-finger domain of ICP0 that disrupt UbcH5a binding has been shown to inhibit ICP0's ability to complement and reactivate HSV-1 mutant viruses in cell culture [44]. Second, biochemical studies of other alphaherpesvirus ICP0 RING-finger orthologs, including BICP0 (bovine), EICP0 (equine) and VICP0 (varicella zoster virus), show equivalent E3 ubiquitin ligase activities in vitro $[45,46]$, which can partially complement the replication of an ICP0-null mutant virus [46].

ICP0 has also been reported to contain a second ubiquitin ligase domain (herpesvirus ubiquitin ligase 1 [HUL-1]) within its C-terminus (Figure 1) [47,48]. This second domain was shown to be required for ICP0 to stimulate the auto-ubiquitination of cdc34 (UBE2RI) in vitro, consequently leading to a differential change in the stability of cyclins D1 and D3 during infection [49]. However, a subsequent report demonstrated that cdc34 protein levels were unaltered during HSV-1 infection and that the degradation of cellular cyclins D1 and D3 occurred in an ICP0-independent manner [50]. Additionally, the HUL-1 region overlaps with several other functional domains of ICP0 (Figure 1), making it difficult to distinguish the role of these domains in ICP0's activities from that proposed for HUL-1. Thus, the role of HUL-1 as an independent ubiquitin ligase domain in ICP0 has yet to be fully established.

One consequence of ICP0's RING-finger ubiquitin ligase activity, as with many ubiquitin ligases, is the propensity for ICP0 to stimulate its own ubiquitination. This activity is readily detected in vitro [42,51] and can be inhibited or reversed by the addition of USP7 to reaction mixtures, as the ubiquitin protease activity of USP7 is capable of cleaving off the ubiquitin chains on ICP0 [51]. Although it is difficult to detect ubiquitinated species of ICP0 during infection, ICP0 USP7-binding domain mutants are comparatively less stable than wild-type ICP0. Likewise, depletion of USP7 by RNA interference (RNAi) has also been shown to decrease the stability and expression levels of ICP0 during infection [51,52]. These data suggest that ICP0 interacts with USP7 in order to protect itself from auto-ubiquitination, thereby enhancing its ability to act as a viral transactivator. Interestingly, ICP0 is capable of catalyzing the ubiquitination of USP7, leading to its proteasomal-dependent degradation [52]. While the reciprocal biochemical activities of ICP0 and USP7 are relatively clear, the biological significance remains to be fully elucidated, as point mutations within ICP0 that disrupt USP7 binding have only a minor impact on lytic viral replication [52]. Nevertheless, these observations may reflect a potential regulatory feedback mechanism that allows ICP0 to modulate its own activity, a phenotype that may become more apparent when low levels of ICP0 are expressed, such as during the initial stages of viral reactivation.

ICP0 has also been shown to directly mediate the ubiquitination of a number of other important cellular proteins, including p53 [53], RNF8 [39] and NF-кB [45], and to stimulate the ubiquitination of PML in transfected cells [54]. Although the exact mechanism(s) by which ICP0 mediates the ubiquitination of PML remains to be determined, SUMOylation of PML plays an important role in ICP0's ability to target PML for proteasomal degradation [54]. Therefore, it is likely that ICP0 employs multiple mechanisms (both direct and indirect) in order to target cellular proteins for ubiquitination. Thus, identifying the cellular substrates and understanding the biochemistry of ICP0 will likely reveal unique insights into how HSV-1 lytic and latent infections are regulated. 
ICP0's E3 ubiquitin ligase activity appears to be regulated by phosphorylation [55]. Tandem mass spectrometric analysis identified three regions of ICP0 (termed Phos 1, 2 and 3) containing a total of 11 putative phosphorylation sites (Figure 1) [56]. Mutation of these sites not only impaired ICP0's ability to transactivate gene expression (specifically in Phos 1 and 3) [56], but also its ubiquitin ligase activity (Phos 1 and 2) [57]. Mutation of Phos 1 inhibited ICP0's ability to conjugate ubiquitin and degrade PML and Sp100 in transfection assays. By contrast, mutation of residues within Phos 2 inhibited ICP0's ability to degrade PML, while retaining its ubiquitin ligase activity. However, analysis of these phosphorylation site mutants in the context of viral infection showed that only mutation of the Phos 1 region had a defect in viral replication in cell culture. Taken together, these data suggest that ICP0 phosphorylation is important for efficient viral replication and establishes a potential mechanism by which ICP0's E3 ubiquitin ligase activity may be regulated through post-translational modifications.

\section{Impairment of the host's antiviral response by ICPO}

Due to the functional importance of ICP0 in the biology of HSV-1 replication, a number of potential mechanisms have been proposed as to how ICP0 works on a molecular level. One established role for ICP0 during viral infection is its ability to inactivate a number of components relating to host cell antiviral defense and stress response pathways, including factors of both intrinsic and innate defenses and one aspect related to acquired immunity [58-62]. Intrinsic defense, in which many core components are also regulated as part of the innate response, consists of pre-existing host cell factors that attempt to silence viral gene expression by making the viral genome functionally inaccessible $[58,59,61,62]$. Initially, we will discuss how ICP0 perturbs three intrinsic defense mechanisms, which are ND10s and their constituent proteins, the chromatinization of the viral genome and the DNA damage response.

ICP0's ability to activate viral gene expression is closely associated with its ability to disrupt ND10s. ND10s are punctate, nuclear suborganelles that contain a variety of nuclear effectors, such as transcription factors, chromatin remodeling factors and DNA damage response members, which collectively participate in a number of important cellular processes, including proliferation, differentiation and antiviral host cell defense (reviewed in [63]). HSV-1 genomes were initially described as being deposited at ND10s [64]; subsequently, ND10-associated proteins have been observed to disassemble and reform at sites associated with incoming viral genomes [65]. The prototypic ND10-associated protein is PML, which nucleates ND10 structures [66]. Other cellular proteins that localize to ND10s include Sp100, hDaxx and ATRX [66-68]. ICP0 inhibits the cumulative repressive effects of various ND10-associated proteins by inducing the disruption of ND10 structures through the degradation of PML, its SUMO-modified isoforms and SUMO-modified Sp100 $[33,35]$, as previously described in this article. This in turn dissociates hDaxx and ATRX away from viral genomes, preventing these ND10-associated proteins from repressing viral gene expression [69]. Determining the contribution of specific ND10 constituent proteins in the biology of HSV-1 replication has primarily been determined by knocking down the expression of these proteins using RNAi technology. Initially, Everett and co-workers showed that cells depleted of PML increased the plaque formation efficiency and gene expression of an ICP0-null mutant virus, but not those of wild-type HSV-1 [70]. Likewise, depletion of Sp100 had a similar effect on plaque formation and gene expression of an ICP0null mutant virus as PML depletion. Combined depletion of both Sp100 and PML synergistically complemented ICP0-null mutant virus replication [71], indicating that ND10associated proteins work cooperatively during intrinsic antiviral defense. Depletion of hDaxx or ATRX also increased ICP0-null mutant virus titers. Notably, exogenous expression of an $\mathrm{hDaxx}$ RNAi resistant form in $\mathrm{hDaxx}$-depleted cells restored the ability of 
this cell line to repress ICP0-null mutant plaque formation, and this repression was dependent on hDaxx's binding partner, ATRX [69]. The fact that an ICP0-null mutant is only partially complemented by depletion of one or more of these ND10-associated factors indicates that other cellular factors, which likely include unidentified ND10 constituent proteins, contribute to the repression of HSV-1 infection, a process that is blocked by the E3 ubiquitin ligase activity of ICP0 during viral infection.

In addition to disrupting ND10s, ICP0 has also been reported to interfere with the chromatinization of viral DNA. During viral infection, a portion of the viral genome rapidly associates with histones, and in the absence of ICP0, these histones take on posttranslational modifications indicative of a repressive chromatin structure. ICP0 has been shown to decrease the amount of histones loaded onto the viral DNA and to promote the acetylation of the histones that remain, resulting in higher levels of viral transcription $[72,73]$. This acetylation of histones directed by ICP0 may be linked to ICP0's ability to bind to the class II histone deacetylases, HDAC-4, -5 and -7 , affecting the suppressive effects of these HDACs [74]. However, additional studies will need to be performed in order to determine whether or not ICP0 has a direct role in viral DNA chromatinization, as opposed to indirectly influencing this phenotype through its ubiquitin ligase activity.

ICP0 has also been proposed to interact with the cellular factor RE1-silencing transcription factor corepressor (CoREST) [75]. It was hypothesized that in the absence of ICP0, CoREST represses HSV-1 transcription and that ICP0 inhibits this process by disrupting the complex comprised of CoREST, REST and histone acetylases by binding to CoREST utilizing sequences within its C-terminus (Figure 1). The proposed repressive role of CoREST and its inactivation by ICP0 were further tested in two subsequent studies. In one report, a CoREST-binding mutant was still capable of efficiently activating viral expression, indicating that this ICP0 domain is largely not required for ICP0's antirepressive function during lytic infection [76]. A second report demonstrated that depletion of CoREST in cell culture did not increase the replication efficiency of an ICP0-null mutant virus [77]. These two reports suggest that ICP0's interaction with CoREST appears not to be involved in ICP0's ability to enhance viral transcription. However, a recent publication has shown that a dominant-negative (dn)-REST expressed from a wild-type HSV-1 genome during infection enhanced viral replication in a mouse ocular model, supporting the hypothesis that a CoREST, REST and histone acetylases complex regulates HSV-1 replication [78]. The role that the ICP0-CoREST interaction plays in ICP0's ability to stimulate viral gene expression in vivo and any indirect affects that dn-REST expression has on the physiology of infected cells remain to be determined.

While perhaps not traditionally thought of as antiviral in nature, the DNA damage response also represents a series of pre-existing cellular factors that can serve to restrict the replication of some viruses. It is unclear as to what exact role the DNA damage response plays during the HSV-1 life cycle; however, it is apparent that ICP0 is involved in regulating many aspects relating to this cellular response. Early during infection, ICP0 appears to initiate damage response pathways by causing activation of the cellular kinase checkpoint kinase 2 (Chk2) in an ATM-dependent manner, resulting in a cell cycle block at the G2/M boundary [79]. Chk2 is required for HSV-1 ICP0-mediated G2/M arrest and enhancement of virus growth. Conversely, ICP0 induces the degradation of another kinase, DNA-PKcs, disrupting the ability of DNA-PKcs to phosphorylate downstream mediator molecules regulating DNA repair in response to double-stranded breaks [30]. Furthermore, ICP0 also targets RNF8 and RNF168 for proteasome-dependent degradation, the activities of which are necessary for maintaining the presence of DNA repair factors at sites of DNA breaks [39]. Each of these actions by ICP0 promotes viral growth, as the loss of Chk2 results in 
lower levels of replication, while a loss in DNA-PKcs or depletion of RNF8 or RNF168 partially compensates the replication defect of an ICP0-null mutant virus.

Besides disrupting or degrading pre-existing cellular defenses, ICP0 is also involved in subverting innate antiviral pathways. Infection by HSV-1 triggers the activation of interferon (IFN) regulatory factor (IRF) 3 , which, in the absence of ICP0, translocates to the nucleus, where it activates the IFN- $\beta$ promoter, the production of IFN- $\beta$ and the initiation of an IFN response [61]. Binding of IFN- $\beta$ to its cognate receptor results in the activation of the transcription factors STAT1 and STAT2, which themselves upregulate a class of genes known as IFN-stimulated genes (ISGs), the products of which have broad antiviral activities. HSV-1 is typically considered to be an IFN-resistant virus, and ICP0 is critical for this resistance. In animal models, ICP0-null mutants are attenuated in both their replication and pathogenesis, while knockout of key IFN-signaling genes such as STAT1 [80] or the IFN- $\alpha / \beta$ receptor [81] can relieve the host's repression of these ICP0 mutants. In cell culture, an HSV-1 mutant that only expresses ICP0 and no other immediate-early genes is able to prevent the upregulation of ISGs initiated by viral infection [82]. At present, the effects of ICP0 on the IFN response appear to be tied to its interaction with IRF3. Following its disruption of intrinsic nuclear defenses, ICP0 is exported to the cytoplasm and, once there, it blocks signaling by IRF3 [83]. While the mechanism is unknown, ICP0 is able to either prevent or reverse the phosphorylation of IRF3 that is necessary for its dimerization, nuclear translocation and target gene activation [83,84].

Concurrent with its disruption of the IFN response, ICP0 prevents the activation of NF- $\mathrm{KB}$ by Toll-like receptors (TLRs) that detect viral components. ICP0 has been reported to mediate a reduction in the levels of the TLR-adaptor proteins MyD88 and Mal, to prevent signaling through TLR-2 [85]. Additionally, ICP0 is able to recruit the normally nuclear USP7 to the cytoplasm [86]. Once in the cytoplasm, USP7 binds to and removes polyubiquitin chains from TRAF6 and IKK $\gamma$, preventing their activation by TLRs and degradation, respectively [86]. Both of these proteins are necessary for the nuclear

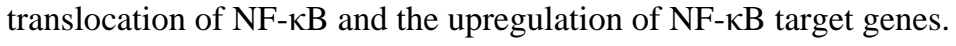

While ICP0 impairs activation of the innate immune response, it is also required for the successful establishment of a lytic infection in cells in which the IFN response has been activated prior to infection. In cells pretreated with type I IFNs (such as IFN- $\beta$ ), ICP0-null mutants can be up to 1000-fold less likely to initiate a lytic infection than wild-type virus [Smith MC \& Davido DJ, Unpublished Data] [87,88].

Expression of ICP0 in trans by either an adenoviral vector or an integrated copy of ICP0 in a cell line can largely ameliorate the inhibitory effects of type I IFNs on the replication of an ICP0-null virus $[87,89]$. Studies performed with mouse embryo fibroblasts derived from knockout mice indicated that PML was primarily responsible for this effect and that the loss of PML could compensate for the lack of ICP0 in IFN-treated cells [90]. However, in PMLdepleted human fibroblast cells, IFN- $\beta$ was still capable of inhibiting the replication of an ICP0-null mutant, although this effect was diminished [91]. This result indicates that other ISGs, in addition to PML, likely play a role in the repression of HSV-1 replication by IFN- $\beta$. Furthermore, a study by Halford and coworkers showed that the acute replication of an ICP0 mutant virus was equally repressed in $P M L$-knockout and wild-type mice, suggesting that the loss of PML (as an ISG) cannot complement the replication of an ICP0 mutant virus in vivo [80]. Although the report that used $P M L$-knockout mouse embryo fibroblasts and the studies that used PML-depleted human fibroblasts and mice in vivo appear to come to different conclusions, PML's role as a mediator of the IFN response may be dependent upon the species of the host, homology between PML orthologs and/or the cell types used in these 
studies. It is still unclear how ICP0 enables HSV-1 to replicate in the presence of an established IFN response.

Lastly, ICP0 has been reported to induce the degradation of the cell surface marker CD83 on mature dendritic cells, which act as antigen-presenting cells [60]. It is hypothesized that the elimination of CD83 from mature dendritic cells prevents or diminishes the stimulation of $\mathrm{T}$ cells, allowing HSV-1 to evade one part of the acquired immune response.

\section{Conclusion \& future perspective}

Virus-host cell interactions determine the outcome (lytic vs latent) of HSV-1 replication. The ubiquitin ligase activity of ICP0 and its ability to stimulate infection tips the scales in favor of viral replication, whether during a primary or recurrent infection, making ICP0 an attractive target for HSV-1 antiviral therapy. ICP0's ability to enhance viral gene expression is mediated by the degradation of cellular 'repressors' and the inactivation of several cellular antiviral responses, which include disrupting ND10 domains, impairing viral DNA chromatinization, altering the host cell's DNA damage response and impairing IRF3 and TLR activation (Figure 3). Most of these functions are dependent upon the E3 ubiquitin ligase activity of ICP0. Ultimately, if novel inhibitors of ICP0's E3 ubiquitin ligase activity, transactivating activity and/or ability to subvert host cell defenses could be identified, these inhibitors would likely maintain the HSV-1 genome in a latent or quiescent state. The crucial benefit derived from such inhibitors is the prevention or attenuation of recurrent HSV-1 diseases. Furthermore, understanding the mechanisms by which these inhibitors function would provide new insights into the roles ICP0 plays during viral infection and in the biology of HSV-1.

\section{Bibliography}

Papers of special note have been highlighted as:

- of interest

-" of considerable interest

1. Cai W, Schaffer PA. Herpes simplex virus type 1 ICP0 regulates expression of immediate-early, early, and late genes in productively infected cells. J Virol. 1992; 66(5):2904-2915. [PubMed: 1313909]

2. Cai WZ, Schaffer PA. Herpes simplex virus type 1 ICP0 plays a critical role in the de novo synthesis of infectious virus following transfection of viral DNA. J Virol. 1989; 63(11):4579-4589. [PubMed: 2552142]

3. Everett RD. Construction and characterization of herpes simplex virus type 1 mutants with defined lesions in immediate early gene 1. J Gen Virol. 1989; 70(Pt 5):1185-1202. [PubMed: 2543774]

4. Sacks WR, Schaffer PA. Deletion mutants in the gene encoding the herpes simplex virus type 1 immediate-early protein ICP0 exhibit impaired growth in cell culture. J Virol. 1987; 61(3):829-839. [PubMed: 3027408]

5. Stow ND, Stow EC. Isolation and characterization of a herpes simplex virus type 1 mutant containing a deletion within the gene encoding the immediate early polypeptide Vmw110. J Gen Virol. 1986; 67(Pt 12):2571-2585. [PubMed: 3025339]

6. Cai W, Astor TL, Liptak LM, Cho C, Coen DM, Schaffer PA. The herpes simplex virus type 1 regulatory protein ICP0 enhances virus replication during acute infection and reactivation from latency. J Virol. 1993; 67(12):7501-7512. [PubMed: 8230470]

7. Halford WP, Schaffer PA. Optimized viral dose and transient immunosuppression enable herpes simplex virus ICP0-null mutants to establish wild-type levels of latency in vivo. J Virol. 2000; 74(13):5957-5967. [PubMed: 10846077] 
8. Leib DA, Coen DM, Bogard CL, et al. Immediate-early regulatory gene mutants define different stages in the establishment and reactivation of herpes simplex virus latency. J Virol. 1989; 63(2): 759-768. [PubMed: 2536101]

9. Halford WP, Schaffer PA. ICP0 is required for efficient reactivation of herpes simplex virus type 1 from neuronal latency. J Virol. 2001; 75(7):3240-3249. [PubMed: 11238850]

10. Everett RD. Trans activation of transcription by herpes virus products: requirement for two HSV-1 immediate-early polypeptides for maximum activity. EMBO J. 1984; 3(13):3135-3141. [PubMed: 6098466]

11. Gelman IH, Silverstein S. Identification of immediate early genes from herpes simplex virus that transactivate the virus thymidine kinase gene. Proc Natl Acad Sci USA. 1985; 82(16):5265-5269. [PubMed: 2991915]

12. O'Hare P, Hayward GS. Three trans-acting regulatory proteins of herpes simplex virus modulate immediate-early gene expression in a pathway involving positive and negative feedback regulation. J Virol. 1985; 56(3):723-733. [PubMed: 2999428]

13. Perry LJ, Rixon FJ, Everett RD, Frame MC, McGeoch DJ. Characterization of the IE110 gene of herpes simplex virus type 1. J Gen Virol. 1986; 67(Pt 11):2365-2380. [PubMed: 3023529]

14. Quinlan MP, Knipe DM. Stimulation of expression of a herpes simplex virus DNA-binding protein by two viral functions. Mol Cell Biol. 1985; 5(5):957-963. [PubMed: 2987684]

15. Everett RD, Maul GG. HSV-1 IE protein Vmw110 causes redistribution of PML. EMBO J. 1994; 13(21):5062-5069. [PubMed: 7957072]

16. Maul GG, Everett RD. The nuclear location of PML, a cellular member of the C3HC4 zinc-binding domain protein family, is rearranged during herpes simplex virus infection by the $\mathrm{C} 3 \mathrm{HC} 4$ viral protein ICP0. J Gen Virol. 1994; 75(Pt 6):1223-1233. [PubMed: 8207389]

17. Chen J, Panagiotidis C, Silverstein S. Multimerization of ICP0, a herpes simplex virus immediateearly protein. J Virol. 1992; 66(9):5598-5602. [PubMed: 1323713]

18. Ciufo DM, Mullen MA, Hayward GS. Identification of a dimerization domain in the C-terminal segment of the IE110 transactivator protein from herpes simplex virus. J Virol. 1994; 68(5):32673282. [PubMed: 8151788]

19. Everett RD, Meredith M, Orr A. The ability of herpes simplex virus type 1 immediate-early protein Vmw110 to bind to a ubiquitin-specific protease contributes to its roles in the activation of gene expression and stimulation of virus replication. J Virol. 1999; 73(1):417-426. [PubMed: 9847347]

20. Barlow PN, Luisi B, Milner A, Elliott M, Everett R. Structure of the C3HC4 domain by $1 \mathrm{H}-$ nuclear magnetic resonance spectroscopy. A new structural class of zinc-finger. J Mol Biol. 1994; 237(2):201-211. [PubMed: 8126734]

21. Everett RD, Barlow P, Milner A, et al. A novel arrangement of zinc-binding residues and secondary structure in the $\mathrm{C} 3 \mathrm{HC} 4$ motif of an alpha herpes virus protein family. J Mol Biol. 1993; 234(4):1038-1047. [PubMed: 8263911]

22. Everett RD. A detailed mutational analysis of Vmw110, a trans-acting transcriptional activator encoded by herpes simplex virus type 1. EMBO J. 1987; 6(7):2069-2076. [PubMed: 2820720]

23. Everett R, O'Hare P, O'Rourke D, Barlow P, Orr A. Point mutations in the herpes simplex virus type 1 Vmw110 RING finger helix affect activation of gene expression, viral growth, and interaction with PML-containing nuclear structures. J Virol. 1995; 69(11):7339-7344. [PubMed: 7474166]

24. Lium EK, Silverstein S. Mutational analysis of the herpes simplex virus type 1 ICP0 C3HC4 zinc ring finger reveals a requirement for ICP0 in the expression of the essential $\alpha 27$ gene. J Virol. 1997; 71(11):8602-8614. [PubMed: 9343218]

25. O'Rourke D, Elliott G, Papworth M, Everett R, O'Hare P. Examination of determinants for intranuclear localization and transactivation within the RING finger of herpes simplex virus type 1 IE110k protein. J Gen Virol. 1998; 79(Pt 3):537-548. [PubMed: 9519832]

26. Harris RA, Everett RD, Zhu XX, Silverstein S, Preston CM. Herpes simplex virus type 1 immediate-early protein Vmw110 reactivates latent herpes simplex virus type 2 in an in vitro latency system. J Virol. 1989; 63(8):5313-5315. 
27. Everett RD, Meredith M, Orr A, Cross A, Kathoria M, Parkinson J. A novel ubiquitin-specific protease is dynamically associated with the PML nuclear domain and binds to a herpesvirus regulatory protein. EMBO J. 1997; 16(7):1519-1530. [PubMed: 9130697]

28. Meredith M, Orr A, Elliott M, Everett R. Separation of sequence requirements for HSV-1 Vmw110 multimerisation and interaction with a 135-kDa cellular protein. Virology. 1995; 209(1):174-187. [PubMed: 7747467]

29. Meredith M, Orr A, Everett R. Herpes simplex virus type 1 immediate-early protein Vmw110 binds strongly and specifically to a 135-kDa cellular protein. Virology. 1994; 200(2):457-469. [PubMed: 8178435]

30. Lees-Miller SP, Long MC, Kilvert MA, Lam V, Rice SA, Spencer CA. Attenuation of DNAdependent protein kinase activity and its catalytic subunit by the herpes simplex virus type 1 transactivator ICP0. J Virol. 1996; 70(11):7471-7477. [PubMed: 8892865]

31. Parkinson J, Lees-Miller SP, Everett RD. Herpes simplex virus type 1 immediate-early protein vmw110 induces the proteasome-dependent degradation of the catalytic subunit of DNAdependent protein kinase. J Virol. 1999; 73(1):650-657. [PubMed: 9847370]

32. Chelbi-Alix MK, de The H. Herpes virus induced proteasome-dependent degradation of the nuclear bodies-associated PML and Sp100 proteins. Oncogene. 1999; 18(4):935-941. [PubMed: 10023669]

33. Everett RD, Freemont P, Saitoh H, et al. The disruption of ND10 during herpes simplex virus infection correlates with the Vmw110- and proteasome-dependent loss of several PML isoforms. $\mathbf{J}$ Virol. 1998; 72(8):6581-6591. [PubMed: 9658103]

34. Muller S, Dejean A. Viral immediate-early proteins abrogate the modification by SUMO-1 of PML and Sp100 proteins, correlating with nuclear body disruption. J Virol. 1999; 73(6):5137-5143. [PubMed: 10233977]

35. Parkinson J, Everett RD. Alphaherpesvirus proteins related to herpes simplex virus type 1 ICP0 affect cellular structures and proteins. J Virol. 2000; 74(21):10006-10017. [PubMed: 11024129]

36. Everett RD, Earnshaw WC, Findlay J, Lomonte P. Specific destruction of kinetochore protein CENP-C and disruption of cell division by herpes simplex virus immediate-early protein Vmw110. EMBO J. 1999; 18(6):1526-1538. [PubMed: 10075924]

37. Lomonte P, Morency E. Centromeric protein CENP-B proteasomal degradation induced by the viral protein ICP0. FEBS Lett. 2007; 581(4):658-662. [PubMed: 17258208]

38-. Lomonte P, Sullivan KF, Everett RD. Degradation of nucleosome-associated centromeric histone H3-like protein CENP-A induced by herpes simplex virus type 1 protein ICP0. J Biol Chem. 2001; 276(8):5829-5835. Paper discovered a link between the DNA damage response and intrinisic host defenses. [PubMed: 11053442]

39. Lilley CE, Chaurushiya MS, Boutell C, et al. A viral E3 ligase targets RNF8 and RNF168 to control histone ubiquitination and DNA damage responses. EMBO J. 2010; 29(5):943-955. [PubMed: 20075863]

40. Everett RD. ICP0 induces the accumulation of colocalizing conjugated ubiquitin. J Virol. 2000; 74(21):9994-10005. [PubMed: 11024128]

41-. Parkinson J, Everett RD. Alphaherpesvirus proteins related to herpes simplex virus type 1 ICP0 induce the formation of colocalizing, conjugated ubiquitin. J Virol. 2001; 75(11):5357-5362. Seminal publication that thoroughly characterized ICP0's E3 ubiquitin ligase activity in vitro. [PubMed: 11333917]

42. Boutell C, Sadis S, Everett RD. Herpes simplex virus type 1 immediate-early protein ICP0 and is isolated RING finger domain act as ubiquitin E3 ligases in vitro. J Virol. 2002; 76(2):841-850. [PubMed: 11752173]

43. Gu H, Roizman B. The degradation of promyelocytic leukemia and Sp100 proteins by herpes simplex virus 1 is mediated by the ubiquitin-conjugating enzyme UbcH5a. Proc Natl Acad Sci USA. 2003; 100(15):8963-8968. [PubMed: 12855769]

44. Vanni, E.; Gatherer, D.; Boutell, C. Functional characterisation of RING finger residues required for ICP0's ability to interact with cellular ubiquitin conjugating enzymes. Presented at: 35 th International Herpesvirus Workshop; UT, USA. 24-30 July 2010; 
45. Diao L, Zhang B, Fan J, et al. Herpes virus proteins ICP0 and BICP0 can activate NF- $\kappa$ B by catalyzing I $\kappa \mathrm{B} \alpha$ ubiquitination. Cell Signal. 2005; 17(2):217-229. [PubMed: 15494213]

46. Everett RD, Boutell C, McNair C, Grant L, Orr A. Comparison of the biological and biochemical activities of several members of the alphaherpesvirus ICP0 family of proteins. J Virol. 2010; 84(7):3476-3487. [PubMed: 20106921]

47. Hagglund R, Van Sant C, Lopez P, Roizman B. Herpes simplex virus 1-infected cell protein 0 contains two E3 ubiquitin ligase sites specific for different E2 ubiquitin-conjugating enzymes. Proc Natl Acad Sci USA. 2002; 99(2):631-636. [PubMed: 11805320]

48. Van Sant C, Hagglund R, Lopez P, Roizman B. The infected cell protein 0 of herpes simplex virus 1 dynamically interacts with proteasomes, binds and activates the cdc34 E2 ubiquitin-conjugating enzyme, and possesses in vitro E3 ubiquitin ligase activity. Proc Natl Acad Sci USA. 2001; 98(15):8815-8820. [PubMed: 11447293]

49. Hagglund R, Roizman B. Herpes simplex virus 1 mutant in which the ICP0 HUL-1 E3 ubiquitin ligase site is disrupted stabilizes cdc34 but degrades D-type cyclins and exhibits diminished neurotoxicity. J Virol. 2003; 77(24):13194-13202. [PubMed: 14645576]

50. Everett RD. Herpes simplex virus type 1 regulatory protein ICP0 does not protect cyclins D1 and D3 from degradation during infection. J Virol. 2004; 78(18):9599-9604. [PubMed: 15331692]

51. Canning M, Boutell C, Parkinson J, Everett RD. A RING finger ubiquitin ligase is protected from autocatalyzed ubiquitination and degradation by binding to ubiquitin-specific protease USP7. J Biol Chem. 2004; 279(37):38160-38168. Established a link between ICP0 and USP7 and their stabilities. [PubMed: 15247261]

52. Boutell C, Canning M, Orr A, Everett RD. Reciprocal activities between herpes simplex virus type 1 regulatory protein ICP0, a ubiquitin E3 ligase, and ubiquitin-specific protease USP7. J Virol. 2005; 79(19):12342-12354. [PubMed: 16160161]

53. Boutell C, Everett RD. The herpes simplex virus type 1 (HSV-1) regulatory protein ICP0 interacts with and ubiquitinates p53. J Biol Chem. 2003; 278(38):36596-36602. [PubMed: 12855695]

54. Boutell C, Orr A, Everett RD. PML residue lysine 160 is required for the degradation of PML induced by herpes simplex virus type 1 regulatory protein ICP0. J Virol. 2003; 77(16):8686-8694. [PubMed: 12885887]

55-. Wilcox KW, Kohn A, Sklyanskaya E, Roizman B. Herpes simplex virus phosphoproteins. I. Phosphate cycles on and off some viral polypeptides and can alter their affinity for DNA. J Virol. 1980; 33(1):167-182. First publication to demonstrate that site-specific post-translational modifications on ICP0 appear to regulate its activities. [PubMed: 6245226]

56. Davido DJ, von Zagorski WF, Lane WS, Schaffer PA. Phosphorylation site mutations affect herpes simplex virus type 1 ICP0 function. J Virol. 2005; 79(2):1232-1243. [PubMed: 15613350]

57. Boutell C, Everett R, Hilliard J, Schaffer P, Orr A, Davido D. Herpes simplex virus type 1 ICP0 phosphorylation mutants impair the E3 ubiquitin ligase activity of ICP0 in a cell type-dependent manner. J Virol. 2008; 82(21):10647-10656. [PubMed: 18715910]

58. Everett RD, Chelbi-Alix MK. PML and PML nuclear bodies: implications in antiviral defence. Biochimie. 2007; 89(6-7):819-830. [PubMed: 17343971]

59. Hagglund R, Roizman B. Role of ICP0 in the strategy of conquest of the host cell by herpes simplex virus 1. J Virol. 2004; 78(5):2169-2178. [PubMed: 14963113]

60. Kummer M, Turza NM, Muhl-Zurbes P, et al. Herpes simplex virus type 1 induces CD83 degradation in mature dendritic cells with immediate-early kinetics via the cellular proteasome. $\mathrm{J}$ Virol. 2007; 81(12):6326-6338. [PubMed: 17428858]

61. Mossman KL, Ashkar AA. Herpesviruses and the innate immune response. Viral Immunol. 2005; 18(2):267-281. [PubMed: 16035939]

62. Weitzman MD, Lilley CE, Chaurushiya MS. Genomes in conflict: maintaining genome integrity during virus infection. Annu Rev Microbiol. 2010; 64:61-81. [PubMed: 20690823]

63. Maul GG, Negorev D, Bell P, Ishov AM. Review: properties and assembly mechanisms of ND10, PML bodies, or PODs. J Struct Biol. 2000; 129(2-3):278-287. [PubMed: 10806078]

64. Maul GG, Ishov AM, Everett RD. Nuclear domain 10 as preexisting potential replication start sites of herpes simplex virus type-1. Virology. 1996; 217(1):67-75. [PubMed: 8599237] 
65. Everett RD, Murray J. ND10 components relocate to sites associated with herpes simplex virus type 1 nucleoprotein complexes during virus infection. J Virol. 2005; 79(8):5078-5089. [PubMed: 15795293]

66. Ishov AM, Sotnikov AG, Negorev D, et al. PML is critical for ND10 formation and recruits the PML-interacting protein daxx to this nuclear structure when modified by SUMO-1. J Cell Biol. 1999; 147(2):221-234. [PubMed: 10525530]

67. Ascoli CA, Maul GG. Identification of a novel nuclear domain. J Cell Biol. 1991; 112(5):785-795. [PubMed: 1999457]

68-. Ishov AM, Vladimirova OV, Maul GG. Heterochromatin and ND10 are cell-cycle regulated and phosphorylation-dependent alternate nuclear sites of the transcription repressor Daxx and SWI/ SNF protein ATRX. J Cell Sci. 2004; 117(Pt 17):3807-3820. Highlights the role of two ND10associated proteins in HSV-1 replication. [PubMed: 15252119]

69. Lukashchuk V, Everett RD. Regulation of ICP0-null mutant herpes simplex virus type 1 infection by ND10 components ATRX and hDaxx. J Virol. 2010; 84(8):4026-4040. [PubMed: 20147399]

70. Everett RD, Rechter S, Papior P, Tavalai N, Stamminger T, Orr A. PML contributes to a cellular mechanism of repression of herpes simplex virus type 1 infection that is inactivated by ICP0. J Virol. 2006; 80(16):7995-8005. [PubMed: 16873256]

71. Everett RD, Parada C, Gripon P, Sirma H, Orr A. Replication of ICP0-null mutant herpes simplex virus type 1 is restricted by both PML and Sp100. J Virol. 2008; 82(6):2661-2672. [PubMed: 18160441]

72. Cliffe AR, Knipe DM. Herpes simplex virus ICP0 promotes both histone removal and acetylation on viral DNA during lytic infection. J Virol. 2008; 82(24):12030-12038. [PubMed: 18842720]

73. Coleman HM, Connor V, Cheng ZS, Grey F, Preston CM, Efstathiou S. Histone modifications associated with herpes simplex virus type 1 genomes during quiescence and following ICP0mediated de-repression. J Gen Virol. 2008; 89(Pt 1):68-77. [PubMed: 18089730]

74. Lomonte P, Thomas J, Texier P, Caron C, Khochbin S, Epstein AL. Functional interaction between class II histone deacetylases and ICP0 of herpes simplex virus type 1. J Virol. 2004; 78(13):67446757. [PubMed: 15194749]

75. Gu H, Roizman B. Herpes simplex virus-infected cell protein 0 blocks the silencing of viral DNA by dissociating histone deacetylases from the CoREST-REST complex. Proc Natl Acad Sci USA. 2007; 104(43):17134-17139. [PubMed: 17939992]

76. Everett RD, Parsy ML, Orr A. Analysis of the functions of herpes simplex virus type 1 regulatory protein ICP0 that are critical for lytic infection and derepression of quiescent viral genomes. J Virol. 2009; 83(10):4963-4977. [PubMed: 19264778]

77. Everett RD. Depletion of CoREST does not improve the replication of ICP0 null mutant herpes simplex virus type 1. J Virol. 2010; 84(7):3695-3698. [PubMed: 20106915]

78. Du T, Zhou G, Khan S, Gu H, Roizman B. Disruption of HDAC/CoREST/REST repressor by dnREST reduces genome silencing and increases virulence of herpes simplex virus. Proc Natl Acad Sci USA. 2010; 107(36):15904-15909. [PubMed: 20798038]

79. Li H, Baskaran R, Krisky DM, et al. Chk2 is required for HSV-1 ICP0-mediated G2/M arrest and enhancement of virus growth. Virology. 2008; 375(1):13-23. [PubMed: 18321553]

80. Halford WP, Weisend C, Grace J, et al. ICP0 antagonizes Stat 1-dependent repression of herpes simplex virus: implications for the regulation of viral latency. Virol J. 2006; 3:44. [PubMed: 16764725]

81. Leib DA, Harrison TE, Laslo KM, Machalek MA, Moorman NJ, Virgin HW. Interferons regulate the phenotype of wild-type and mutant herpes simplex viruses in vivo. J Exp Med. 1999; 189(4): 663-672. [PubMed: 9989981]

82. Eidson KM, Hobbs WE, Manning BJ, Carlson P, DeLuca NA. Expression of herpes simplex virus ICP0 inhibits the induction of interferon-stimulated genes by viral infection. J Virol. 2002; 76(5): 2180-2191. [PubMed: 11836395]

83. Paladino P, Collins SE, Mossman KL. Cellular localization of the herpes simplex virus ICP0 protein dictates its ability to block IRF3-mediated innate immune responses. PLoS ONE. 2010; 5(4):E10428. [PubMed: 20454685] 
84. Melroe GT, DeLuca NA, Knipe DM. Herpes simplex virus 1 has multiple mechanisms for blocking virus-induced interferon production. J Virol. 2004; 78(16):8411-8420. [PubMed: 15280450]

85. van Lint AL, Murawski MR, Goodbody RE, et al. Herpes simplex virus immediate-early ICP0 protein inhibits Toll-like receptor 2-dependent inflammatory responses and NF-kappaB signaling. J Virol. 2010; 84(20):10802-10811. [PubMed: 20686034]

86. Daubeuf S, Singh D, Tan Y, et al. HSV ICP0 recruits USP7 to modulate TLR-mediated innate response. Blood. 2009; 113(14):3264-3275. [PubMed: 18952891]

87. Harle P, Sainz B Jr, Carr DJ, Halford WP. The immediate-early protein, ICP0, is essential for the resistance of herpes simplex virus to interferon- $\alpha / \beta$. Virology. 2002; 293(2):295-304. [PubMed: 11886249]

88. Mossman KL, Saffran HA, Smiley JR. Herpes simplex virus ICP0 mutants are hypersensitive to interferon. J Virol. 2000; 74(4):2052-2056. [PubMed: 10644380]

89. Everett RD, Orr A. Herpes simplex virus type 1 regulatory protein ICP0 aids infection in cells with a preinduced interferon response but does not impede interferon-induced gene induction. J Virol. 2009; 83(10):4978-4983. [PubMed: 19264774]

90. Chee AV, Lopez P, Pandolfi PP, Roizman B. Promyelocytic leukemia protein mediates interferonbased anti-herpes simplex virus 1 effects. J Virol. 2003; 77(12):7101-7105. [PubMed: 12768029]

91. Everett RD, Young DF, Randall RE, Orr A. STAT-1- and IRF-3-dependent pathways are not essential for repression of ICP0-null mutant herpes simplex virus type 1 in human fibroblasts. J Virol. 2008; 82(17):8871-8881. [PubMed: 18579584] 
Executive summary

\section{Infected cell protein 0: a viral transactivator}

- Structure-function studies have identified domains in infected cell protein 0 (ICP0), located in its $\mathrm{N}$ - and $\mathrm{C}$-termini, that contribute to its transcriptional activation of viral genes.

\section{The E3 ubiquitin ligase activity of ICPO}

- The RING-finger motif of ICP0 is required for efficient viral replication and its E3 ubiquitin ligase activity.

- Several cellular targets of ICP0-directed degradation regulate its biological functions.

- The stability of ICP0 is controlled by its interactions with USP7 and autoubiquitination.

- Phosphorylation of ICP0 appears to modulate its transactivating, nuclear domain (ND)10-disrupting, and E3 ubiquitin ligase activities.

\section{Impairment of the host's antiviral response by ICP0}

- ICP0 impairs intrinsic defenses by several mechanisms, promoting viral replication.

- ICP0 impairs the activation of the interferon response, mediates resistance to a pre-existing interferon response and impairs a component of acquired immunity.

\section{Future perspective}

- ICP0 plays a pivotal role in the HSV-1 life cycle, making it an attractive target for designing anti-HSV-1 drugs or therapies.

- Inhibitors of ICP0's E3 ubiquitin ligase activity, transactivating activity and/or ability to subvert host cell defenses will likely be effective in preventing recurrent HSV-1 diseases. 


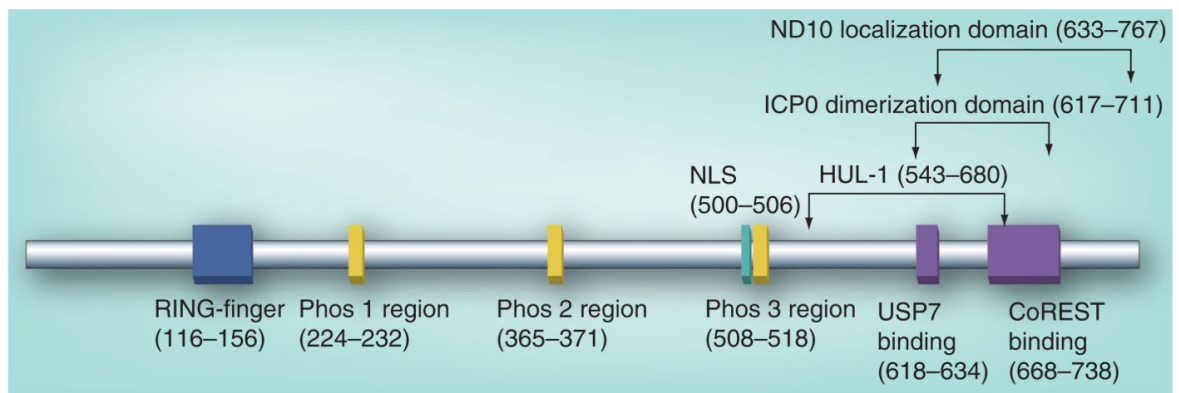

Figure 1. ICP0 domains

The functional domains of ICP0, which include the RING-finger motif, regions of phosphorylation (Phos 1-3), NLS, USP7-, ICP0-, CoREST-binding, ND10 localization and HUL-1 domains. The amino acid positions for each domain are indicated in parentheses. CoREST: RE1-silencing transcription factor corepressor; HUL: Herpesvirus ubiquitin ligase; ICP0: Infected cell protein 0; ND10: Nuclear domain 10; NLS: Nuclear localization signal; RING: Really interesting new gene; USP: Ubiquitin-specific protease. 


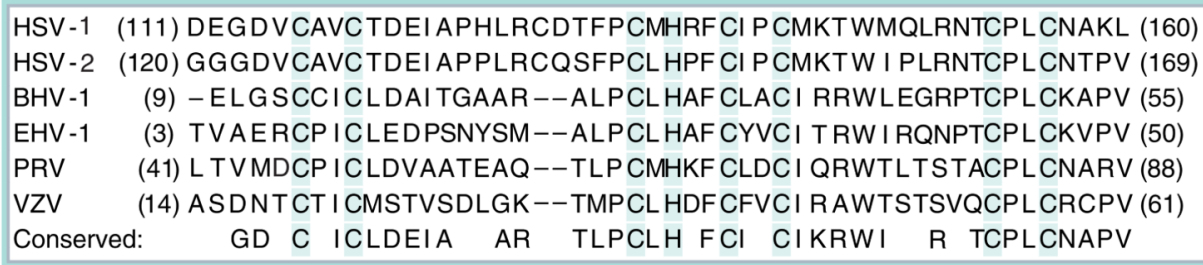

Figure 2. Amino acid alignment of ortholog ICP0 RING-finger domains from HSV-1, HSV-2, bovine herpes virus type-1, equine herpes virus type-1, pseudorabies virus and varicella zoster virus

Gray vertical bars highlight conserved cysteine and histidine residues within the $\mathrm{C} 3 \mathrm{HC} 4$ RING domain that co-ordinate the binding of two zinc atoms. Bracketed numbers refer to the amino acid positions of the first and last amino acid in each alignment with respect to their parental polypeptide sequences.

ICP0: Infected cell protein 0; RING: Really interesting new gene. 


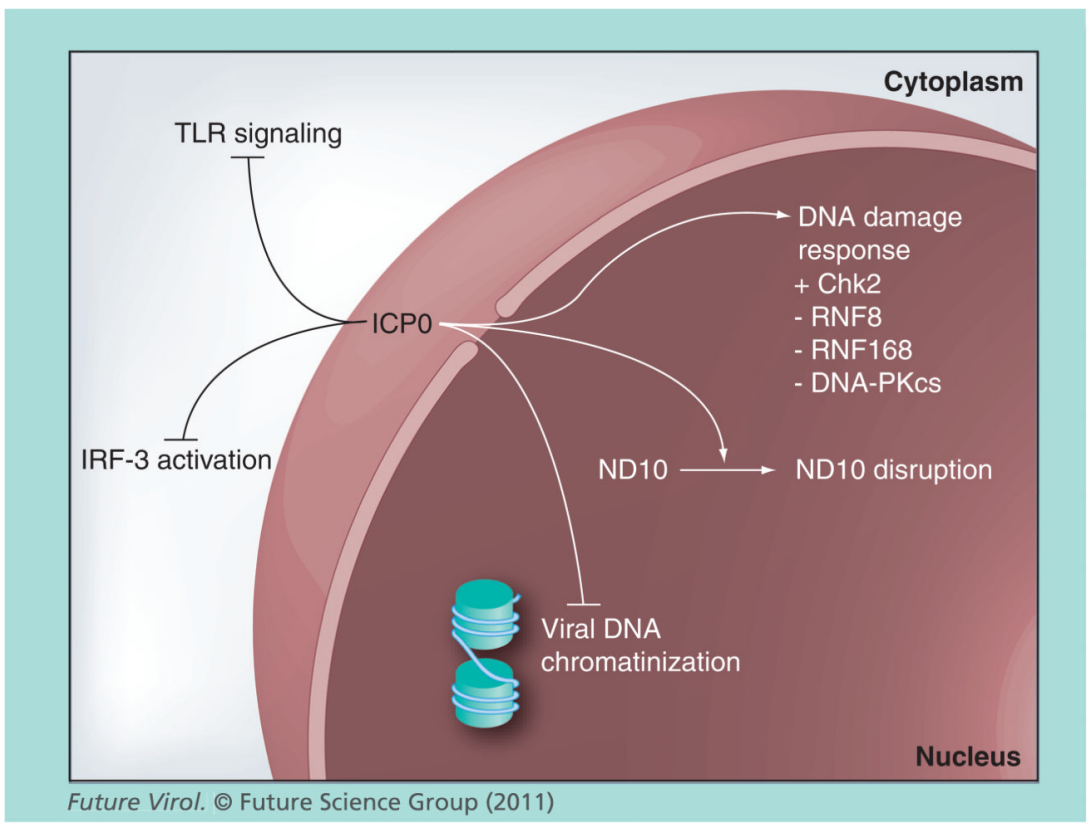

Figure 3. Cellular pathways modulated by ICP0 that enhance viral replication ICP0 inhibits or impairs IRF3 activation, TLR signaling and the chromatinization of viral DNA and disrupts ND10. For the DNA damage response, ICP0 directly or indirectly activates (+) Chk2 or inhibits (-) RNF8, RNF168 and DNA-PKcs.

Chk2: Checkpoint kinase 2; DNA-PKcs: DNA-dependent protein kinase; ICP0: Infected cell protein 0; IRF: Interferon regulatory factor; ND10: Nuclear domain 10; RNF: RING-finger; TLR: Toll-like receptor. 